\title{
THE DEPENDENCE OF REVERSE RECOVERY TIME ON BARRIER CAPACITANCE AND SERIES-ON RESISTANCE IN SCHOTTKY DIODES
}

\author{
OLEKSANDR VEHER, NATALJA SLEPTSUK, JANA TOOMPUU, OLEG KOROLKOV \\ \& TOOMAS RANG \\ Department of Electronics, Tallinn University of Technology, Estonia
}

\begin{abstract}
It is well known that reverse recovery time $\left(\tau_{\text {rr }}\right)$ is important when a diode is used in a switching application. It is the time taken to switch the diode from its forward conducting or 'ON' state to the reverse 'OFF' state. As a result, there is reverse current overshoot when switching from the forward conducting state to the reverse blocking state. The time is needed to remove the reverse recovery charge (Qrr) as a Schottky diode is normally measured in nanoseconds, ns. Some diodes exhibit $\tau_{\mathrm{rr}}$ of $100 \mathrm{p} / \mathrm{s}$. The experimental part consists of the measurements of the reverse recovery time on a special tester - LEMSYS DMS dynamic parameter system - the SiC Schottky diodes C3D10060A are used. In the present work, measuring the results of reverse recovery time depends on additional resistors and condensers connected to the initial diode. It has been shown that the recovery time is mostly determined by the barrier capacitance, and should not depend on the resistance of the base.

Keywords: reverse recovery time, Schottky diode, barrier capacitance, series-on resistance.
\end{abstract}

\section{INTRODUCTION}

The theoretical advantages of $\mathrm{SiC}$ technology are obvious. It is well known that $\mathrm{SiC}$ diodes are the perfect choice in high efficiency, high-voltage applications such as switch-mode power supplies and high-speed inverters. Nowadays, the new technology promises new semiconductor products with a behavior very close to ideal parameters such as forward voltage drops, high operating temperature, ultra low Qrr, low leakage current. The remaining advantage for $\mathrm{SiC}$ technology is its ideal dynamic behavior [1]. The low recovery current is the key benefit of using SiC Schottky diodes. When the diode switches from its forward conducting or ' $\mathrm{ON}$ ' state to the reverse 'OFF', the total charge that must be removed during reverse recovery is denoted by $\mathrm{Q}_{\mathrm{RR}}$, i.e. the reverse recovery charge, which leads to an appearance of the reverse current that causes additional loss (switching loss) in the diode (eqn 1):

$$
\tau_{r r} \cong \sqrt{\frac{2 Q_{R R}}{d i / d t}}
$$

When the diode has been conducting, a charge is stored at the junction and the bulk of the semiconductor. With reference to Fig. 1, when the diode current becomes negative, the diode continues to conduct. This reverse conduction continues until the negative current sweeps away the charge stored in the junction. At that moment, the diode gains its reverse blocking capability and its voltage charges to the reverse recovery voltage. The time needed for the diode to gain blocking capability is denoted by $t_{a}$. An additional time, $t_{b}$, is needed to remove the stored charge from the bulk of the semiconductor. Therefore, the total turn-off time is the reverse recovery time of the diode, $\tau_{\mathrm{rr}}=\mathrm{t}_{\mathrm{a}}+\mathrm{t}_{\mathrm{b}}$. The total charge that must be removed during reverse recovery is denoted by $\mathrm{Q}_{\mathrm{RR}}$, i.e. the reverse recovery charge. The peak negative current during reverse recovery is denoted by $\mathrm{I}_{\mathrm{R} R}$. Fig. 1 provides the relations applied to the diode turn-off. The use of additional load of capacitance and/ or resistance must lead to the change in reverse recovery time and current $\mathrm{I}_{\mathrm{rr}}$. 


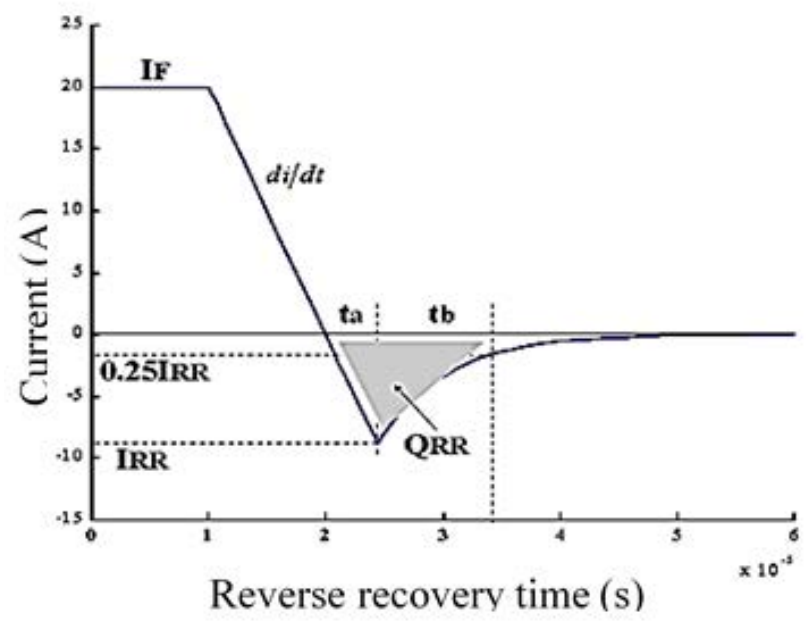

Figure 1: Current during diode turn-off [2].

The aim of this work was to determine which of the key parameters, series-on resistance and/or barrier capacitance should be taken into account when producing the SiC Schottky diodes for use in extreme operating conditions such as radiation rich environments, high frequency etc.

\section{MATERIALS AND METHODOLOGY}

$\mathrm{SiC}$ Schottky diodes that were used for the experiment were commercial purchased Cree SiC Schottky power diodes 600V - C3D10060A. We conducted experiments with different commercial stationary condensers and resistors, connected in series and in parallel to the diode one by one.

To carry out the electrical measurements, we used the following equipment:

- an Agilent B1500A semiconductor device analyser

- a LEMSYS DMS dynamic parameter system

- $\quad$ the generator AFG 3252

- Tektronix

- an Agilent MSOS104A oscilloscope.

Capacitance-voltage $(\mathrm{C}-\mathrm{V})$ and current-voltage (I-V) measurements of the diode with different resistors and condensers values have been measured and collected I-V and C-V are presented. Reverse recovery time dependences of the initial diode and diode connected in series and in parallel with different resistors and condensers have been measured. In addition, the measurements result of combined resistor and condenser together connected to the initial diode have been made and shown.

\section{RESULTS AND DISCUSSION}

Fig. 2 shows the typical views of current-voltage (I-V) characteristics at room temperature for the initial diode and diode with different resistors. The values of resistors used for the experiment are the following: $150 \mathrm{~m} \Omega, 220 \mathrm{~m} \Omega, 270 \mathrm{~m} \Omega, 330 \mathrm{~m} \Omega, 390 \mathrm{~m} \Omega, 470 \mathrm{~m} \Omega, 510 \mathrm{~m} \Omega$ 
and $560 \mathrm{~m} \Omega$. All resistors were metal oxide film resistors with rated output of $1 \mathrm{~W}$ and rated voltage of $350 \mathrm{~V}$.

As shown in Fig. 2, the I-V exhibits a quite good diode behaviour with different resistor values. The value of series resistance $\mathrm{R}_{\mathrm{S}}$ is growing with the increasing of the resistor value connected to the diode. The series-on resistance of a Schottky diode is the sum of the barrier resistance, the resistance due to the epi layer and the resistance due to the substrate. Fig. 3 shows the linear dependence of $\mathrm{R}_{\mathrm{S}}$ on the different values of resistors.

As expected the $\mathrm{C}-\mathrm{V}$ characteristics at room temperature for the initial diode and diode with different resistors showed no changes. The same results were for the I-V characteristics at room temperature for the initial diode and the diode with connected condensers.

Fig. 4 shows the typical $\mathrm{C}-\mathrm{V}$ dependence at room temperature for the same experiment conditions as for I-V measurements. The condensers used for the experiment were ceramic with the following capacitance: $100 \mathrm{pF}, 180 \mathrm{pF}, 270 \mathrm{pF}, 330 \mathrm{pF}$ and $510 \mathrm{pF}$.

As seen from Fig. 4 the capacitance-voltage characteristics are displaced parallel to themselves with the different values of condensers connected to the initial diode.

The typical reverse recovery characteristics for the initial Schottky diode is shown in Fig. 5.

The measurements of the reverse recovery time for the initial diode with resistor or condenser have been made as well. For the demonstration of the results, we have chosen one resistor of $270 \mathrm{~m} \Omega$ and one condenser of $378 \mathrm{pF}$ for the reverse recovery time characteristics Fig. 6.

Additionally, combining some resistors together with condensers connected to the initial diode for the measurement of the reverse recovery time are shown in Fig. 7.

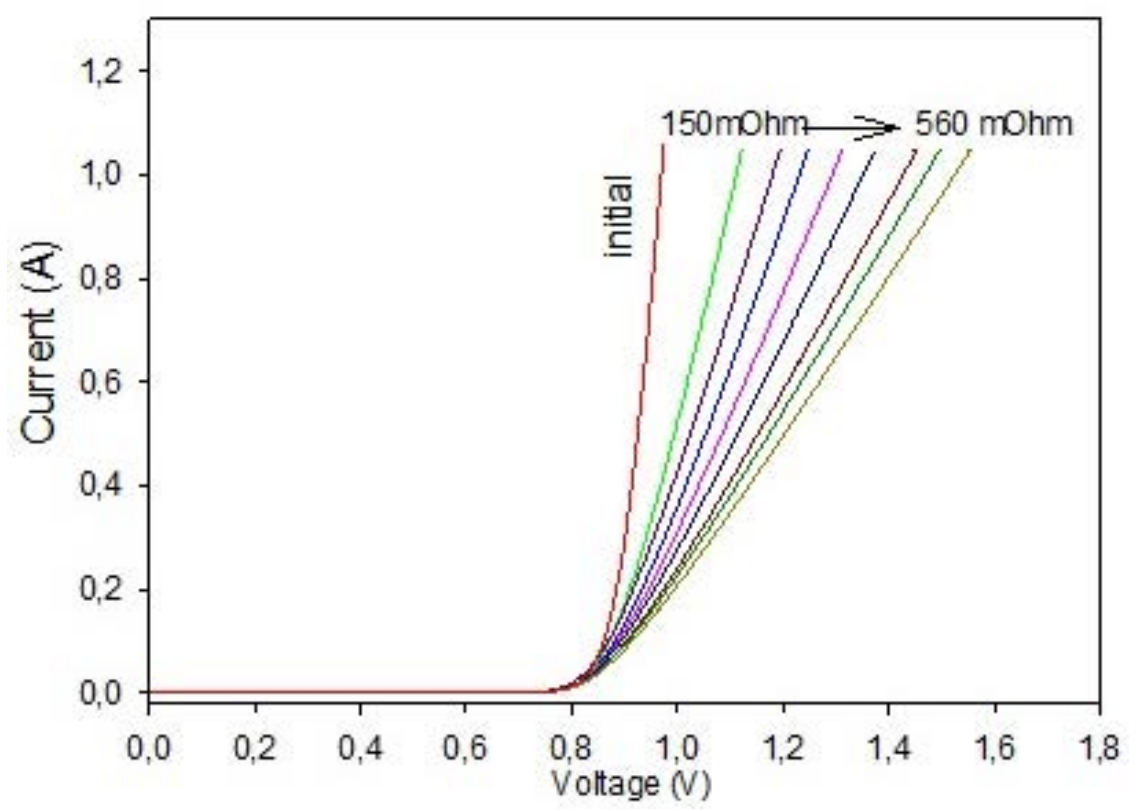

Figure 2: Current-voltage characteristics for the initial diode and diode measured with different resistors values (from $150 \mathrm{~m} \Omega$ to $560 \mathrm{~m} \Omega$ ). 


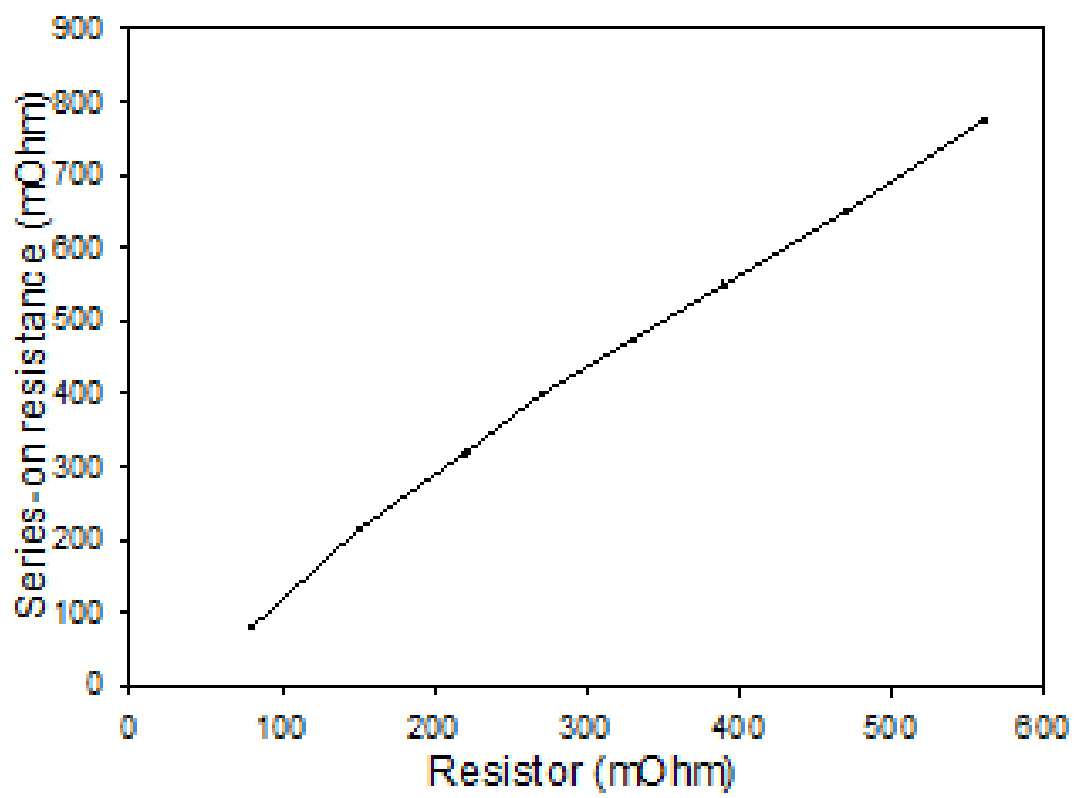

Figure 3: Series-on resistance dependence on different resistor values.

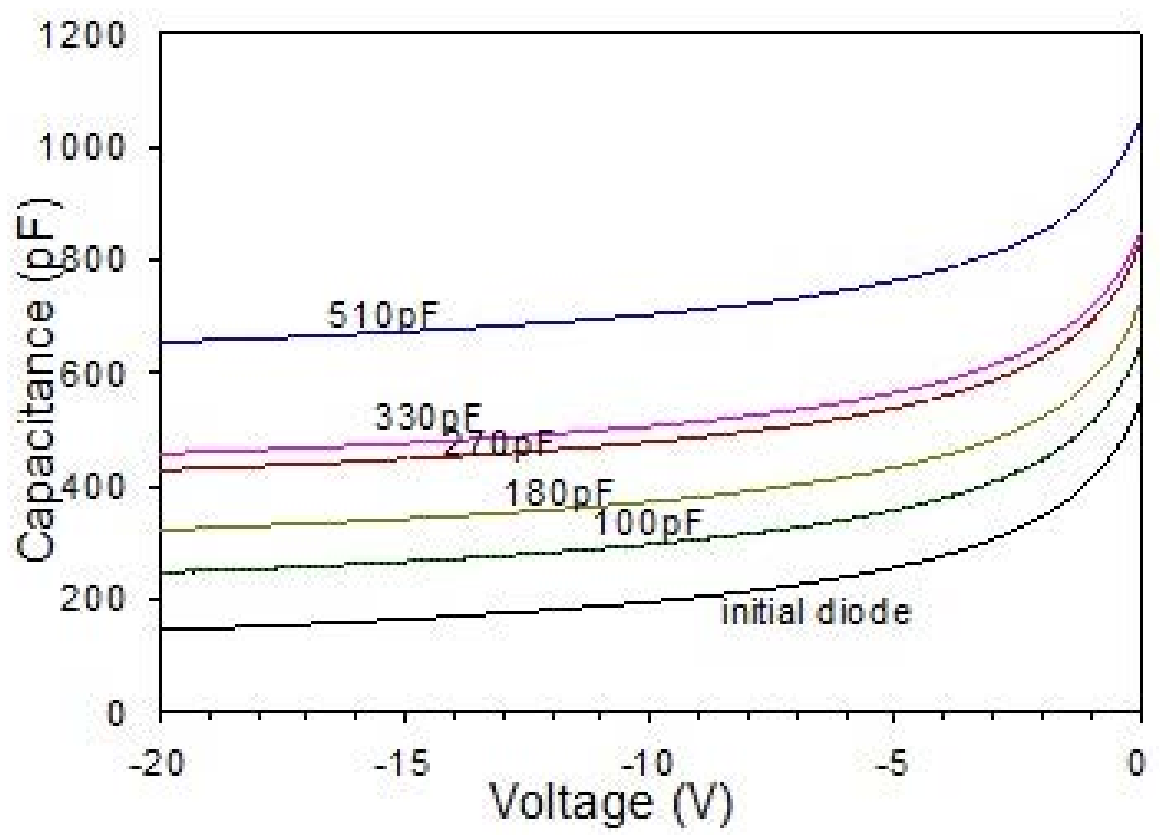

Figure 4: Capacitance-voltage characteristics for the initial diode and diode with different condensers in parallel (from $100 \mathrm{pF}$ to $510 \mathrm{pF}$ ). 


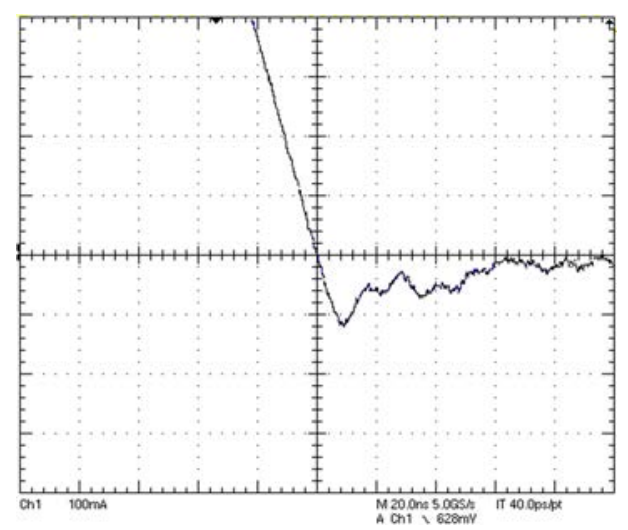

Figure 5: Reverse recovery time characteristic for the initial Schottky diode (I $=100 \mathrm{~mA} / \operatorname{div}, \tau=20 \mathrm{~ns} / \operatorname{div})$.

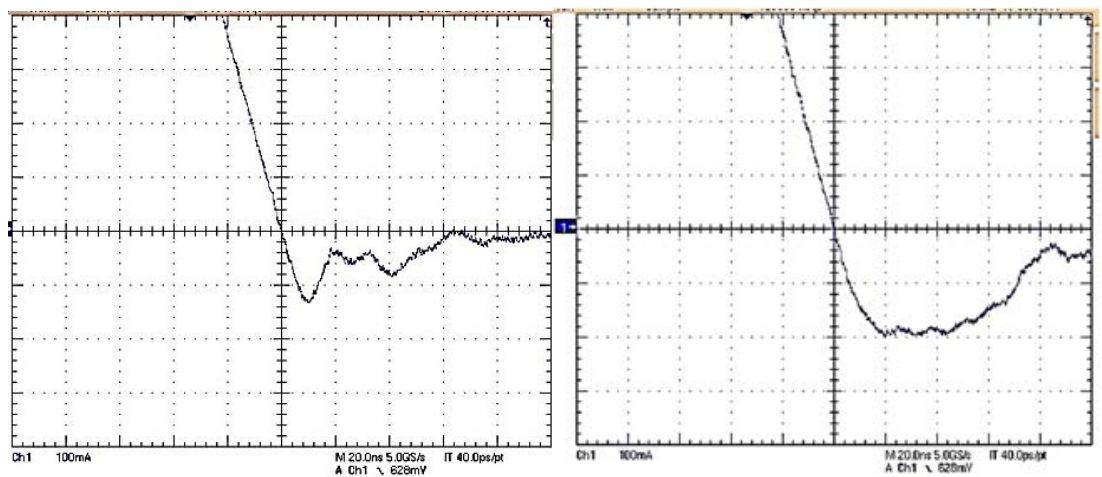

Figure 6: Reverse recovery time for the diode with resistor $(270 \mathrm{~m} \Omega)$ on the left and for the diode with condenser ( $378 \mathrm{pF})$ on the right (I=100mA/div, $\tau=20 \mathrm{~ns} / \mathrm{div})$.

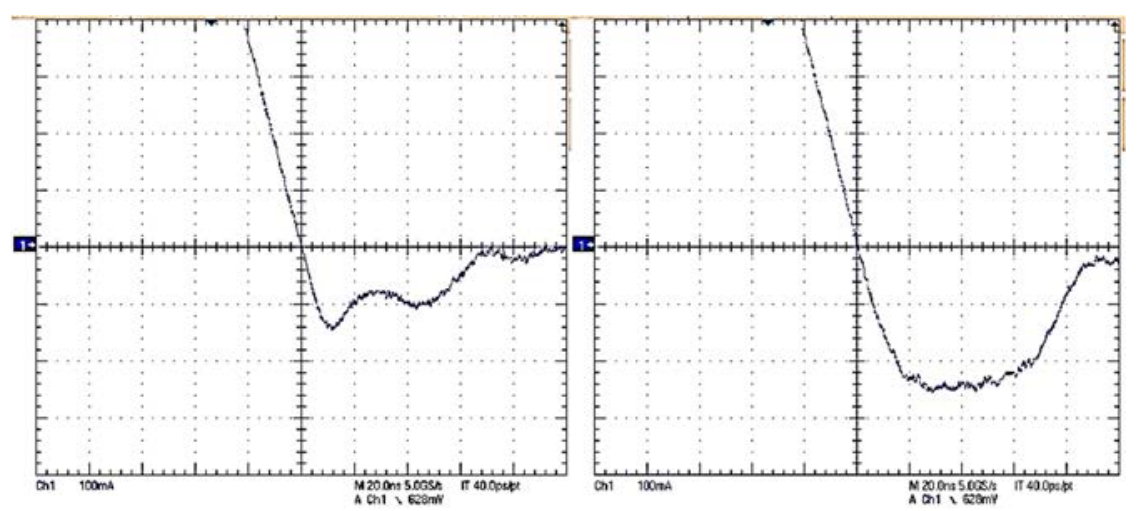

Figure 7: Reverse recovery time for the diode with condenser and resistor $(100 \mathrm{pF}+560$ $\mathrm{m} \Omega)$ on the left and for the diode with condenser and resistor $(378 \mathrm{pF}+330 \mathrm{~m} \Omega)$ on the right $(\mathrm{I}=100 \mathrm{~mA} / \mathrm{div}, \tau=20 \mathrm{~ns} / \mathrm{div})$. 


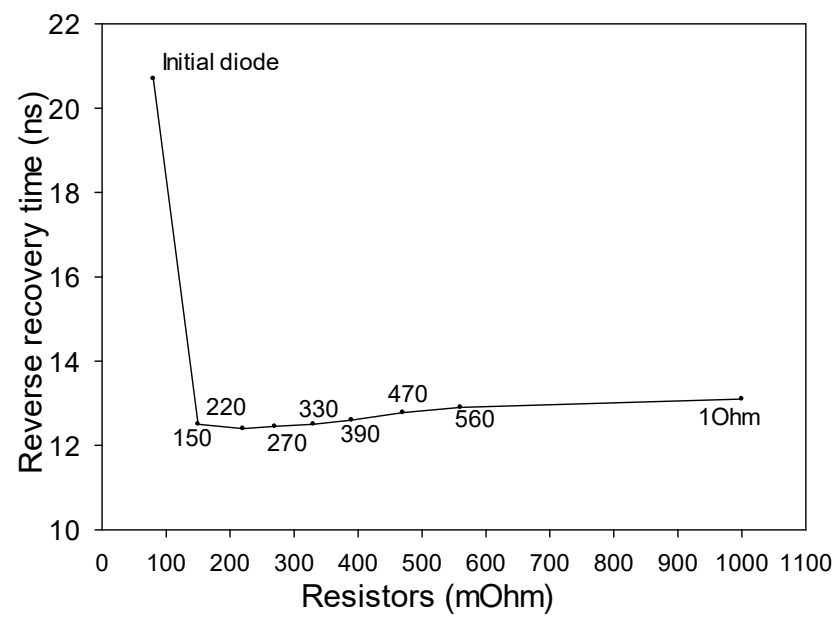

Figure 8: Reverse recovery time dependence on the initial diode and different values of resistors connected to the diode one by one.

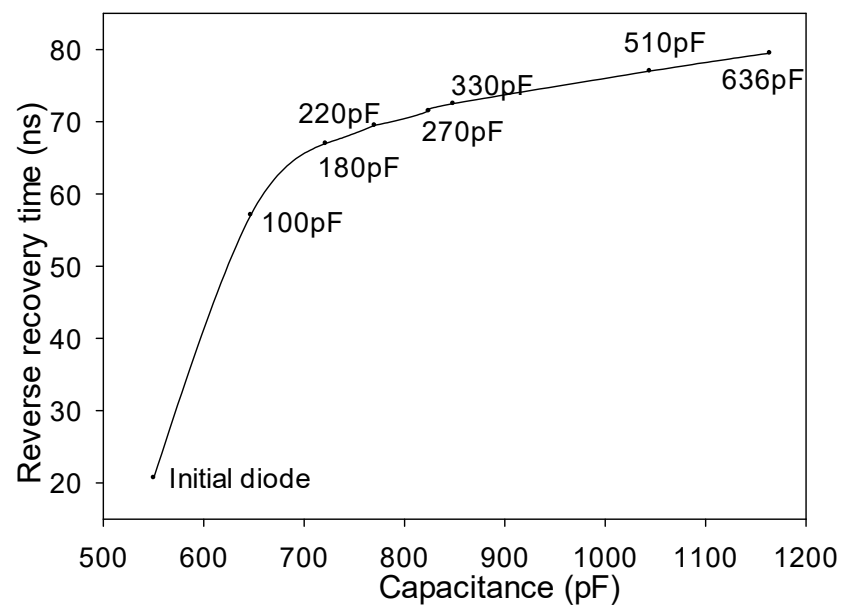

Figure 9: Reverse recovery time dependence on different values of condensers connected to the diode one by one.

The reverse recovery time dependences with all used values of condensers and resistors shown in Figs 8 and 9. As seen from the Fig. 8 the reverse recovery time drops sharply after the connecting the first resistor and then remains independent on the value of the resistors. As for the Fig. 9, we can see the increasing of the $\tau_{\text {rr }}$ more than 3 times depending on the capacitance values. This can be easily explained if we imagine the Schottky as a parallel-plate capacitor [3]. The larger value of condenser, the larger the space charge between parallel-plate capacitors, the more time it takes for the diode to remove the total charge from the junction region when reverse recovered. 
When combining the resistors and condensers connected to the initial diode, one may note that reverse recovery time is determined by the capacitance of the additional condenser and is independent on the additional resistance (Fig. 10).

The idea of carrying out the work presented in this publication appeared during the research of the degradation of $600 \mathrm{~V}, 4 \mathrm{H}-\mathrm{SiC}$ Schottky diodes under irradiation with $0.9 \mathrm{MeV}$ electrons [4]. Among other characteristics studied during the work, the reverse recovery time $\left(\tau_{\mathrm{rr}}\right)$ was analyzed as a function of the radiation dose. To explain the variation of $\tau_{\text {rr }}$ with increasing radiation density, the following version was proposed. It is known that the recharge time of barrier capacitance $\left(\tau_{M}\right)$ between the two equilibrium states mostly determined by the Maxwell relaxation time expression (eqn 2):

$$
\tau_{M}=\rho \varepsilon \varepsilon_{0},
$$

where $\rho$ is the resistivity, $\varepsilon$ the dielectric constant, and $\varepsilon_{0}$ the electric constant. Rewriting the expression as $\tau_{M}=R_{S} C_{0} \varepsilon \varepsilon_{0}$, where $R_{S}$ is series on resistance and $C_{0}$ - barrier capacitance at zero bias, we can see that reverse recovery time $\left(\tau_{r r}\right)$ determined by $\tau_{M}$ is equal to $R_{S} C_{0}$. Thus, the change in the reverse recovery time will be determined by the influence of two mutually compensating processes. Specifically, the barrier capacitance becomes lower due to the decrease in the $\mathrm{N}_{\mathrm{d}}-\mathrm{N}_{\mathrm{a}}$ concentration, on the one hand, and to the increase in $R_{S}$ because of the decrease in the number of carriers and in their mobility, on the other hand. The latter occurs because the carrier lifetime becomes shorter due to the scattering on additional radiation defects. As a result, the decrease in $\tau_{r r}$ at low irradiation doses, when the increase in $R_{s}$ is not significant yet, is determined by the decrease in $C_{0}$. At doses more than $2 \times 10^{16} \mathrm{~cm}^{-2}$, the key influence on the variation of $\tau_{\mathrm{rr}}$ will be exerted by the increase in $R_{s}$ and ohmic resistance of n-base.

Since substrate resistance can be considered as an external resistance with respect to the metal-semiconductor junction, and the barrier capacitance can be simplified to a plate capacitor, these quantities can be modulated by external resistors and capacitors. This is what exactly has been done in this paper. Since the results are perfectly illustrated by the graphs presented above, it is possible to make a reliable conclusion that the recovery time is mostly determined by the barrier capacitance and should not depend on the resistance of the base. The initial sharp drop of $\tau_{r r}$ when connecting an external resistor can be explained by the instrumental specificity of the measurements. The turn-off curve configuration changes. The $\mathrm{Q}_{\mathrm{RR}}$ charge remains the same due to the elongation of the $t_{\mathrm{b}}$ branch, but this does not take into account the measurement method (see Figs 1, 6, 7).

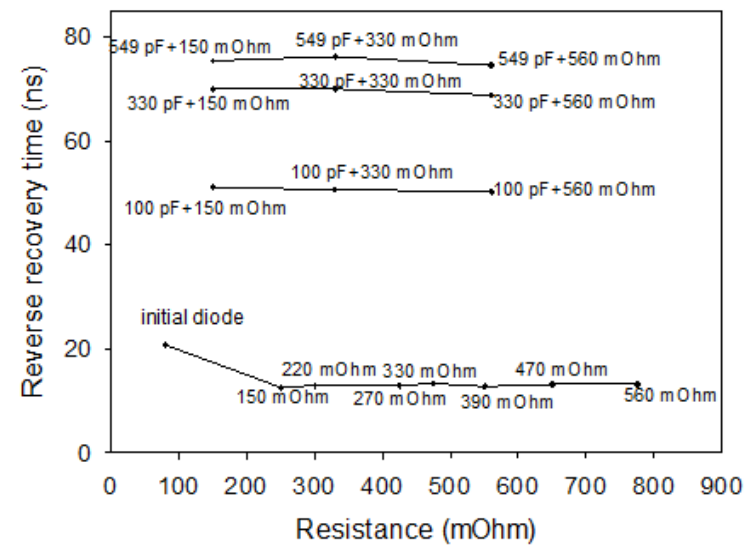

Figure 10: Reverse recovery time dependence on combined resistors and condensers. 


\section{SUMMARY}

The modelling of the diode operation in extreme conditions such as radiation rich environments, high frequency etc. is very important. Conducted experiments with additional resistors and condensers connected to the initial commercial Schottky diode presented here showed that additional capacitance increases reverse recovery time dramatically while additional resistance decreases $\tau_{\mathrm{rr}}$ by 1.5 times. The reverse recovery time as well as the junction capacitance are very important in high-frequency operation of the diode.

\section{ACKNOWLEDGEMENTS}

This project has received funding from the European Union's Horizon 2020 research and innovation programme under ERA chair Project COEL grant (No 668995), and from the Estonian Research Council through the Institutional Research Project IUT19-11. This study was partly supported by PUT 1435 .

\section{REFERENCES}

[1] Hodge S., Cree Inc. 2004, Online. http://www.powerelectronics.com/content/sicschottky-diodes-power-factor-correction.

[2] Experiment 1: switching characteristics of power diodes, Online. http://howard.engr.siu.edu/staff1/hatz/EE483/LABS/Exp1.html. Accessed on: 1 Apr. 2017.

[3] Cory, R., Schottky diodes. MPDIGEST, Skyworks Solutions, Inc., pp. 1-5, 2009.

[4] Lebedev, A.A., Davydovskaya, K.S., Kozlovski, V.V., Korolkov, O., Sleptsuk, N. \& Toompuu, J., Degradation of $600-\mathrm{V}$ 4H-SiC Schottky diodes under irradiation with $0.9 \mathrm{MeV}$ electrons. Presented at the ECSCRM2016 (European Conference on Silicon Carbide and Related Materials), Halkidiki, Greece, 2016. 